\title{
Network and Service Management
}

\section{George Pavlou and Aiko Pras}

This is the fifth issue of the series on Network and Service Management that is published twice a year, in April and October. The series provides articles on the latest developments in this well established discipline, highlighting recent research achievements and providing insight into both theoretical and practical issues related to the evolution of the discipline from different perspectives. The series provides a forum for the publication of both academic and industrial research, addressing the state of the art, theory, and practice in network and service management.

In the last editorial we mentioned that the recently established IEEE Electronic Transactions on Network and Service Management (eTNSM) journal had passed key IEEE approval stages, becoming a full-fledged IEEE transactions journal in January 2007. So far the journal has published two issues per year (http://www.comsoc.org/eTNSM). The editorial of the second quarter 2006 issue states the following: "In the past few years we have witnessed a significant growth in the area of network and service management yet at the same time we lacked a corresponding presence and visible venue in IEEE to publish high-quality papers in this area. eTNSM was established by the Communications Society in 2004 as an introductory initiative for timely electronic presentation of archival research on the management of networks, systems, services and applications, as well as on issues in communications software, service engineering, policies and business processes for network and service management. The success of the eTNSM trial encouraged the initiative toward a proposal for an IEEE transactions journal in this area. In June 2006, a new journal titled IEEE Transactions on Network and Service Management (TNSM) has gone through the last phase approval by the IEEE and will start as a full fledge IEEE Transactions in January 2007. This is an exciting event for the systems research community at large and for the network and service management community in particular!"

Another important development for the community was, at the end of June 2006, the completion of one and a half years of integration work in the European EMANICS project on Management of the Internet and Complex Services. This is an EU Network of Excellence that brings together 13 research institutions active in the area of network and service management. It encompasses work areas dealing with integration (long-term vision, virtual laboratory, and testbeds), dissemination (a new European conference discussed later, electronic dissemination, training and technology transfer, open source initiatives), and joint research activities (scalable, economic and autonomic management). For more information, visit the project site http://www.emanics.org/. You will also find there a newsletter with community news, events, and developments, which is published three times a year. The network had its first yearly evaluation in March 2007 and the evaluation outcome was very positive. In all four categories (integration, dissemination, research, and project management) the network received a very positive evaluation and relevant comments from the reviewers. The presentations used for the evaluation are also available on the network's public Web site.

It is also worth noting that one of the key annual events in this area, Manweek 2007, is to be held 29 October to 2 November, 2007 in San Jose, California, and includes among other events the 18th IFIP/IEEE International Workshop on Distributed Systems: Operations and Management (DSOM 2007) and the 10th IFIP/IEEE International Conference on Management of Multimedia and Mobile Networks and Services (MMNS 2007). The other key annual event is the IEEE/IFIP Network Operations and Management Symposium (NOMS 2008), which will take place 7-11 April 2008 in Salvador, Bahia, Brazil. NOMS together with IM constitute the premier annual event in network and service management.

We again experienced an overwhelming interest in the fourth issue, receiving 16 submissions. All these submissions were subject to three independent reviews, and we finally selected four articles, resulting in a 25 percent acceptance rate. Reviews for the first article, in which the second series editor, Dr. Pras, is a leading co-author, were independently handled by the first series editor, Prof. Pavlou. We intend to maintain our rigorous review process in future issues, thus maintaining the high quality of published articles.

The first article, "Key Research Challenges in Network Management" by Pras, Schoenwaelder, Burgess, Festor, Martinez Perez, Stadler, and Stiller, is a presentation of the outcome of discussions that took place during a workshop that looked at future key challenges in network management. The presented challenges fall under the following areas: management architectures, scalable distributed monitoring, data analysis and visualization, ontologies, economic aspects of management, probabilistic approaches, and the behavior of managed systems.

The second article, "Toward Autonomic Management of Communications Networks" by Jennings, Van der Meer, Balasubramaniam, Botvich, Ó Foghlú, Donnelly, and Strassner, discusses autonomic principles in managing communication networks and uses information and ontological modeling to capture knowledge relating to network capabilities, constraints, and business policies together with reasoning and learning techniques to enhance and evolve this knowledge. Network elements are automatically configured in response to context changes, closing the autonomic loop.

The third and fourth articles address complementary topics in the general area of home networks, gateways, and service provisioning. The third article, "Multiservice Home Gateways: Business Model, Execution Environment, Management Infrastructure" by Royon and Frenot, presents first an overview of home gateway environments, addressing execution environments and management solutions. It then discusses new requirements emerging from 
the multiservice model and presents the implementation of a multiservice gateway together with relevant evaluation results.

Finally, the fourth article, "Local and Remote Management Integration for Flexible Service Provisioning to the Home" by Nikolaidis, Papastefanos, Doumenis, Stassinopoulos, and Drakos, addresses the problem of increased costs of customer relationship management due to parameter misconfiguration in customer premises equipment (CPE). It proposes a solution that allows an operator to access the CPE within a home network through a bridging residential gateway using the DSL Forum CPE WAN management protocol as well as universal plug and play. A relevant prototype implementation is presented and evaluated.

We hope that readers of this issue again find the articles informative, and we will endeavor to continue with similar issues in the future. We would finally like to thank all the authors who submitted articles to this series and the reviewers for their valuable feedback and comments on the articles.

\section{Biographies}

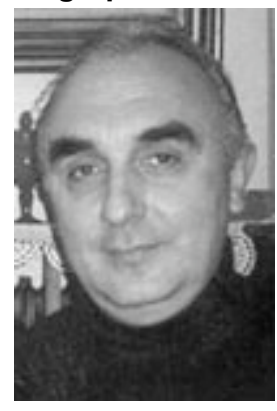

GEORGE PAVLOU is a professor of communication and information systems at the Center for Communication Systems Research, Department of Electrical Engineering, University of Surrey, United Kingdom, where he leads the activities of the Networks Research Group. He received a Diploma in electrical and mechanical engineering from the National Technical University of Athens, Greece, and M.Sc. and Ph.D. degrees in computer science from University College London, United Kingdom. His research interests focus on network management, networking, and service engineering, including policy-based management, programmable networks, traffic engineering, multimedia service control, and object-oriented communications middleware. He has been instrumental in a number of European and U.K. research projects, and has contributed to standardization activities in ISO, ITU-T, and IETF. He was Technical Program Co-Chair of the Seventh IFIP/IEEE Integrated Management Symposium (IM 2001).

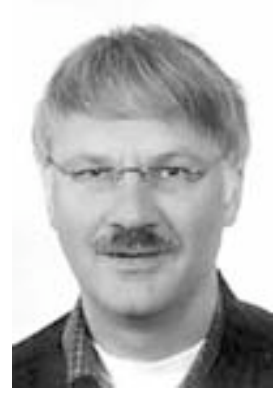

AIKO PRAS is an associate professor in the Departments of Electrical Engineering and Computer Science at the University of Twente, The Netherlands, and a member of the Design and Analysis of Communication Systems Group. He received a Ph.D. degree from the same university for his thesis, Network Management Architectures. His research interests include network management technologies, Web services, network measurements, and accounting. He has participated in many European and Dutch research projects, such as SURFnet6 RoN, M2C, WASP, and Internet NG. He is currently a research leader in the European Network of Excellence on Management of the Internet and Complex Services (EMANICS). He has also contributed to research and standardization activities as a member of the Internet Research Task Force (IRTF) Network Management Research Group (NMRG). He was Technical Program Co-Chair of the Ninth IFIP/IEEE Integrated Management Symposium (IM 2005). 